\title{
Intensifying renal replacement therapy during pregnancy: the role for nocturnal home hemodialysis
}

\author{
Kelly L. Craig • Tiina Podymow $\cdot$ Robert P. Pauly
}

Received: 6 November 2009/Accepted: 9 November 2009/Published online: 24 November 2009

(C) Springer Science+Business Media, B.V. 2009

\begin{abstract}
Fertility among women receiving conventional hemodialysis or peritoneal dialysis is very low. For those able to conceive it appears that infant survival is poor, and prematurity and its related complications are still commonplace. Nocturnal hemodialysis (NHD) is a form of intensive, selfadministered hemodialysis whereby patients receive 3-4 times the duration of conventional hemodialysis resulting in superior removal of uremic toxins compared to traditional dialysis modalities. NHD has been associated with increased fertility, infants with higher birth weights born at more advanced gestational ages, and fewer maternal and fetal complications. These encouraging results suggest a greater role for much more intensive dialysis in pregnancy.
\end{abstract}

Keywords Nocturnal hemodialysis .

Pregnancy

K. L. Craig · R. P. Pauly $(\bowtie)$

Department of Medicine, Division of Nephrology and Transplantation Immunology, University of Alberta, 11-107 Clinical Sciences Building, 8440, 112th Street, Edmonton, AB T6G 2G3, Canada

e-mail: robert.pauly@ualberta.ca

T. Podymow

Department of Medicine, Division of Nephrology, McGill University Health Centre, McGill University, Montreal, QC, Canada

\section{Commentary}

Pregnant women with end-stage renal disease (ESRD) pose a unique and anxiety provoking challenge to nephrologists and obstetricians alike because pregnancy for dialysis-dependent women remains a relatively rare occurrence and because fetal outcomes have been historically poor. There are few data quantifying fertility among women receiving dialysis. In a registry of 6,230 women in the United States between the ages of 14 and 44 years receiving either hemodialysis or peritoneal dialysis between 1992 and 1996, pregnancy was observed in only $2 \%$ [1]. A Belgian study reported an incidence of pregnancy of 0.3 per hundred patient-years for 1,472 women between the ages of 18 and 44 from 1975 to 1996 [2]. Other studies are more difficult to interpret due to incomplete sampling of disparate source populations, and an inconsistent method of defining fertility. Fetal outcomes of these rare pregnancies are also poor with successful deliveries observed in $40-50 \%$ of cases based on registry or large survey data [1-3]. Only $0-16 \%$ of pregnancies progressed to full term ( $\geq 37$ weeks gestation), and many resulted in birth at $<32$ weeks gestation amid complications such as low birth weights, infantile respiratory distress syndrome, retinopathy of prematurity, patent ductus arteriosus, intraventricular hemorrhage, to name just a few [1-4].

International Urology and Nephrology has been an ongoing forum for discussing pregnancy-related 
issues in ESRD. A report by Gumus et al. [5] explored the relationship between glomerular filtration rate and parity and found that pregnancyassociated hyperfiltration did not adversely affect kidney function in women with multiple pregnancies. A recent report by Smith and colleagues highlighted the utility of intensifying the peritoneal dialysis prescription to include 24-h continuous cycler peritoneal dialysis during the third trimester [6], and in the current issue, Tuot et al. [7] present an interesting case of nutritional support provided to a pregnant woman on hemodialysis with hyperemesis gravidarum using intradialytic parenteral nutrition (IDPN). The latter two reports underscore the need for increased dialysis dose to mitigate the deleterious effects of the uremic milieu on the developing fetus, a practice that is in agreement with expert opinion [8-10]. Indeed, a recent retrospective study of $28 \%$ women receiving hemodialysis found that birth weight $\geq 1,500 \mathrm{~g}$ and a gestational age $\geq 32$ weeks correlated with maternal blood urea nitrogen levels of $<49$ and $<48 \mathrm{mg} / \mathrm{dL}$, respectively (18 and $17 \mathrm{mmol} / \mathrm{L}$ ) [4]. This report provides objective evidence supporting the traditional recommendation that dialysis be intensified during pregnancy to achieve a target urea concentration $<50 \mathrm{mg} / \mathrm{dL}$.

The degree to which renal replacement therapy is to be augmented in order to achieve this goal is relatively arbitrary with consensus opinion suggesting that weekly hemodialysis time be increased to at least 20-24 h [8-10]. A systematic evaluation of longer weekly treatment on pregnancy outcomes was not available until recently. Barua et al. [11] at the University of Toronto described their outcomes in a cohort of pregnant women receiving nocturnal home hemodialysis (NHD). NHD is an intensive form of renal replacement therapy whereby patients selfadminister their hemodialysis on 4-6 nights per week with each session lasting 6-8 h; thus patients typically receive 3-4 times the amount of hemodialysis as those undergoing conventional thrice weekly treatments [12]. The Toronto experience from 2001 to 2006 describes 7 pregnancies among 45 women of childbearing age (14-44 years) receiving NHD at the time of conception. Thus, $15.6 \%$ of their cohort became pregnant, a proportion substantially greater than previous reports. The mean weekly duration of NHD at the time of conception was $36 \pm 10 \mathrm{~h}$, and this was further intensified to $48 \pm 5 \mathrm{~h}$ after pregnancy was confirmed. One pregnancy was electively terminated during the first trimester and all but one of the remaining pregnancies progressed to $\geq 36$ weeks with a mean birth weight of $2,418 \mathrm{~g}$. Three infants were born by spontaneous vaginal deliveries, one was induced and required vacuum extraction and two necessitated delivery by Caesarean section. One fetus had evidence of intrauterine growth restriction and was delivered at 36 weeks weighing $2,020 \mathrm{~g}$, while another was small for gestational age (at the 20th percentile) and delivered at 30 weeks; no other fetal complications were reported. With respect to the course of dialysis during pregnancy, all women had systolic and diastolic blood pressures within the physiologic range appropriate for stage of gestation; two women required treatment with either low-dose methyldopa or low-dose labetolol. Hemoglobin was managed with increasing doses of erythropoietin and supplemental iron. Due to the long duration of each treatment session and the associated removal of phosphate, the dialysate was supplemented with sodium phosphate in all cases. Mean pre-dialysis urea concentrations ranged from 21 to $39 \mathrm{mg} / \mathrm{dL}$ (7.6-14.0 mmol/L) while post-dialysis urea concentrations ranged from 3 to $9 \mathrm{mg} / \mathrm{dL}(1.0-3.2 \mathrm{mmol} / \mathrm{L})$.

This case series of six successful pregnancies among prevalent NHD patients are remarkable in many ways. Firstly, the fertility of women receiving this intensive form of renal replacement therapy appears to be substantially greater than previously reported. Though the $15.6 \%$ proportion of women conceiving while on NHD may well be an overestimation (since spontaneous abortions prior to clinical confirmation of pregnancy remain unknown) this limitation is true for all other previous reports of fertility statistics and should not obscure the significance of this finding. The reason for increased fertility is unknown though presumably related to improved clearance of uremic toxins normalizing the hypothalamic-pituitary-ovarian axis abnormalities of ESRD [13]. Secondly, NHD patients achieved higher birth weights and pregnancy duration was longer, a marked improvement from previously published literature. Finally, maternal-fetal complications associated with kidney disease, such as intrauterine growth restriction, maternal hypertension and polyhydramnios, were fewer or not observed. This, too, may be due to minimizing the uremic environment 
and optimizing fluid balance management. The authors concede that a major limitation to their case series is the small sample size and the absence of an appropriate control group. However, due to the rarity of pregnancy among dialysis patients, historical controls from the published literature is not only reasonable but the only realistic approach. Hopefully, outcomes in larger numbers of NHD patients will support this initially positive report.

Notwithstanding the limitations of the Toronto case series, the NHD experience has important implications for women with ESRD wishing to conceive or who are already pregnant. In view of the traditionally poor maternal-fetal outcomes of pregnancy, conception for women on dialysis cannot be encouraged based solely on the results of this single-case series. However, for those women adamantly wishing to become pregnant (and who are not imminently transplantable), a significantly more intensive dialysis regimen should be considered. Additionally, while all women of childbearing age receiving dialysis therapy should be counseled regarding contraception, this is especially true for those receiving NHD, whose fertility appears to be much higher than for women receiving conventional dialysis. Finally, women with ESRD who do become pregnant routinely have their dialysis dose intensified from the usual $12 \mathrm{~h}$ per week to greater than $20 \mathrm{~h}$ per week in accordance with expert opinion. The Toronto experience suggests that weekly dialysis duration $>40 \mathrm{~h}$ is associated with improved outcomes. Though only a few centers have NHD programs, even a conventional hemodialysis unit might consider offering prolonged daily dialysis (e.g., 6 weekly sessions lasting $8 \mathrm{~h}$ per session) using a dialysis prescription similar to NHD. Such a time-consuming commitment to dialysis therapy may seem overwhelming, but the prospect of improved maternal-fetal outcomes may prove to be the necessary motivation for expecting mothers.

Hopefully, the encouraging outcomes with NHD will prompt consideration of this dialytic paradigm for pregnant women with ESRD by their nephrology and high-risk obstetrical team. Broader uptake of NHD for this purpose and additional reports of pregnancy outcomes will better delineate the risks and benefits of NHD in this setting compared to traditional dialysis modalities.

\section{References}

1. Okundaye I, Abrinko P, Hou S (1998) Registry of pregnancy in dialysis patients. Am J Kidney Dis 31:766-773

2. Bagon JA, Vernaeve H, De Muylder X et al (1998) Pregnancy and dialysis. Am J Kidney Dis 31:756-765

3. Toma H, Tanabe K, Tokumoto T et al (1999) Pregnancy in women receiving renal dialysis or transplantation in Japan: a nationwide survey. Nephrol Dial Transplant 14:15111516

4. Asamiya Y, Otsubo S, Matsuda Y et al (2009) The importance of low blood urea nitrogen levels in pregnant patients undergoing hemodialysis to optimize birth weight and gestational age. Kidney Int 75:1217-1222

5. Gumus, II, Uz E, Bavbek N et al (2009) Does glomerular hyperfiltration in pregnancy damage the kidney in women with more parities? Int Urol Nephrol 41(4):927-932

6. Smith WT, Darbari S, Kwan M et al (2005) Pregnancy in peritoneal dialysis: a case report and review of adequacy and outcomes. Int Urol Nephrol 37:145-151

7. Tuot D, Gibson S, Caughey A et al (2009) Intradialytic hyperalimentation as adjuvant support in pregnant hemodialysis patients: case report and review of the literature. Int Urol Nephrol. doi 10.1007/s11255-009-9671-5

8. Hou S (2004) Daily dialysis in pregnancy. Hemodial Int $8: 167-171$

9. Reddy SS, Holley JL (2007) Management of the pregnant chronic dialysis patient. Adv Chronic Kidney Dis 14:146155

10. Haase M, Morgera S, Bamberg C et al (2005) A systematic approach to managing pregnant dialysis patients-the importance of an intensified haemodiafiltration protocol. Nephrol Dial Transplant 20:2537-2542

11. Barua M, Hladunewich M, Keunen J et al (2008) Successful pregnancies on nocturnal home hemodialysis. Clin J Am Soc Nephrol 3:392-396

12. Pauly RP, Chan CT (2007) Reversing the risk factor paradox: is daily nocturnal hemodialysis the solution? Semin Dial 20:539-543

13. Palmer BF (1999) Sexual dysfunction in uremia. J Am Soc Nephrol 10:1381-1388 